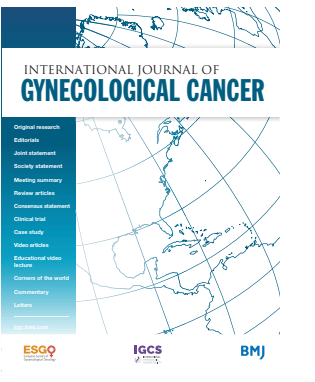

For numbered affiliations see end of article.

Correspondence to Dr Gwenael Ferron, Department of Surgical Oncology, Institut Claudius Regaud Institut Universitaire du Cancer de Toulouse - Oncopole, 1 Avenue Irène Joliot-Curie 31059 , Toulouse Cedex 9, France; ferron.gwenael@iuct-oncopole. fr

Accepted 6 July 2020 Published Online First 13 August 2020

\title{
Vaginal reconstruction after pelvic exenteration with a vertical deep inferior epigastric perforator flap in 10 steps
}

Martina Aida Angeles, ${ }^{1}$ Carlos Martínez-Gómez (D) ,, ${ }^{1,2}$ Alejandra Martinez (D) , ${ }^{1,2}$ Thomas Meresse, ${ }^{3}$ Dimitri Gangloff, ${ }^{3}$ Gwenael Ferron ${ }^{1,4}$

\section{ABSTRACT}

Pelvic exenteration is a radical and challenging surgery requiring a functional reconstruction after pelvic organ resection and, to date, it remains the only treatment with curative intent in selected cases of gynecological cancer relapse previously treated with radiotherapy. Organ replacement including vaginal reconstruction has evolved parallel to the refinement of surgical resection and represents a major goal. The advantages of vaginal reconstruction after pelvic exenteration include anatomical and functional reconstruction which would optionally allow sexual intercourse, better patients' quality of life, self-esteem, and body image, and lower long-term postoperative complications related to 'empty pelvis syndrome'. ${ }^{1}$ Among the different techniques described for vaginal reconstruction, the vertical rectus abdominis myocutaneous (VRAM) flap represents the most commonly employed technique due to its surgical simplicity and the low rate of immediate postoperative complications. ${ }^{2}$ The main constraint of this technique is related to fascia and muscle harvesting, which can compromise the abdominal wall integrity in the long term. ${ }^{3}$ The deep inferior epigastric perforator (DIEP) flap was described for vaginal reconstruction as a technique which allowed the sparing of the muscle and the fascia from the abdominal wall, ${ }^{4}$ hence, reducing the rate of donor site complications. ${ }^{3}$ In addition, this technique does not require vascular anastomoses and, therefore, the risk of thrombosis is low. ${ }^{1}$ In this video 1 , vaginal reconstruction with the DIEP flap has been standardized in the 10 following steps:

Step 1: Patient selection

Step 2: Preoperative doppler perforator identification

Step 3: Midline incision

Step 4: Perforator artery dissection

Step 5: Skin flap harvesting

Step 6: Anterior rectus abdominis muscle fascia opening

Step 7: Transmuscular flap transposition

Step 8: Inferior epigastric artery skeletonization

Step 9: Flap conformation for vaginal reconstruction
Check for updates

(C) IGCS and ESGO 2020. No commercial re-use. See rights and permissions. Published by BMJ.

To cite: Angeles MA Martínez-Gómez C, Martinez A et al. Int J Gynecol Cancer 2020;30:2015-2016.

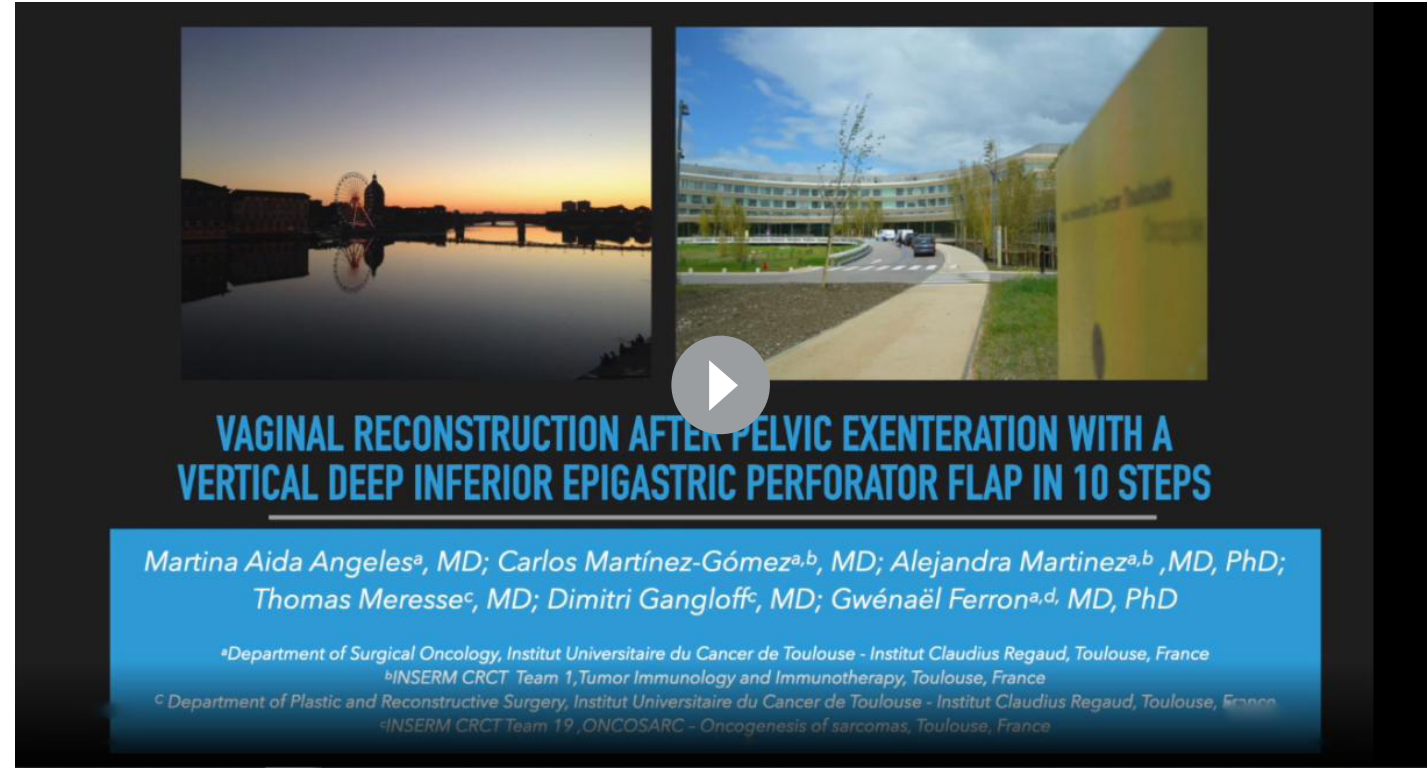

Video 1. Vaginal reconstruction after pelvic exenteration with a vertical Deep Inferior Epigastric Perforator flap 


\section{Video article}

\section{Step 10: Postoperative care}

The sequences correspond to different patients who underwent an anterior or total pelvic exenteration followed by complex pelvic and urinary reconstruction for gynecologic malignancies.

To summarize, the DIEP pedicled flap for vaginal reconstruction represents a good alternative to the VRAM flap since it minimizes donor site complications and its elasticity is higher. ${ }^{3}$ However, DIEP harvesting demands a long training and high-level surgical skills, requiring a multidisciplinary surgical team including oncologic reconstructive surgeons.

\section{Author affiliations}

${ }^{1}$ Department of Surgical Oncology, Institut Claudius Regaud - Institut Universitaire du Cancer de Toulouse - Oncopole, Toulouse, France

${ }^{2}$ INSERM CRCT Team 1, Tumor Immunology and Immunotherapy, Toulouse, France ${ }^{3}$ Department of Plastic and Reconstructive Surgery, Institut Claudius Regaud Institut Universitaire du Cancer de Toulouse - Oncopole, Toulouse, France

${ }^{4}$ INSERM CRCT Team 19, ONCOSARC - Oncogenesis of sarcomas, Toulouse, France

Twitter Martina Aida Angeles @AngelesFite and Alejandra Martinez @Alejandra

Contributors MAA: conceptualization, video editing, writing original draft. CM-G: conceptualization, video editing, writing original draft. AM: conceptualization, project administration, supervision, writing review. TM: conceptualization, project administration, surgery and video recording supervision, writing review. DG: conceptualization, project administration, surgery and video recording supervision, writing review. GF: conceptualization, project administration, surgery and video recording, supervision, writing review.
Funding Martina Aida Angeles acknowledges the grant support from "la Caixa" Foundation, Barcelona (Spain), ID 100010434. The fellowship code is LCF/BQ/ EU18/11650038.

Competing interests GF reports personal fees from Olympus outside of the submitted work.

Patient consent for publication Not required.

Provenance and peer review Not commissioned; externally peer reviewed.

Data availability statement All data relevant to the study are included in the article

\section{ORCID iDs}

Carlos Martínez-Gómez http://orcid.org/0000-0002-9652-7880

Alejandra Martinez http://orcid.org/0000-0002-7633-3536

\section{REFERENCES}

1 Ferron G, Gangloff D, Querleu D, et al. Vaginal reconstruction with pedicled vertical deep inferior epigastric perforator flap (DIEP) after pelvic exenteration: a consecutive case series. Gynecol Oncol 2015;138:603-8.

2 Horch RE, Hohenberger W, Eweida A, et al. A hundred patients with vertical rectus abdominis myocutaneous (VRAM) flap for pelvic reconstruction after total pelvic exenteration. Int $J$ Colorectal Dis 2014;29:813-23.

3 Qiu SS, Jurado M, Hontanilla B. Comparison of TRAM versus DIEP flap in total vaginal reconstruction after pelvic exenteration. Plast Reconstr Surg 2013;132:1020e-7.

4 Wang X, Qiao Q, Burd A, et al. A new technique of vaginal reconstruction with the deep inferior epigastric perforator flap: a preliminary report. Plast Reconstr Surg 2007;119:1785-90. 